\title{
Inflammatory response and insulin signaling alteration induced by PCB77
}

\author{
Jing Wang ${ }^{1, *}$, Xiaowen $\mathrm{Lv}^{2}$, Yuguo $\mathrm{Du}^{1}$ \\ 1. State Key Laboratory of Environmental Chemistry and Ecotoxicology, Research Center for Eco-Environmental Sciences, \\ Chinese Academy of Sciences, Beijing 100085, China.E-mail: wangjing@rcees.ac.cn \\ 2. Feed Safety Reference Laboratory of Ministry of Agriculture, Feed Research Institute, \\ Chinese Academy of Agricultural Sciences, Beijing 100081, China
}

Received 16 September 2009; revised 09 December 2009; accepted 18 December 2009

\begin{abstract}
A specific mechanism whereby inflammation may contribute to cardiovascular diseases (CVD), insulin resistance (IR) and type II diabetes is the induction of endothelial dysfunction placing the vascular endothelium in a key unifying position for the shared pathogenesis of these diseases. However, the mechanisms by which PCBs induce endothelial cell dysfunction are not clearly understood. In the present study, we used human umbilical vascular endothelial cells (HUVEC) as model, and inflammatory response and insulin signaling alteration induced by PCBs were examined. Results showed that PCB77 induced the expression of proinflammatory cytokines including IL-6 and TNF $\alpha$ and induced U937 adhesion to HUVEC cells consistent with increased NFKB transcription activity. On the other hand, PCB77 blocked insulin-activated Akt signaling pathway, which was restored by pretreatment with TNF $\alpha$ neutralization antibody. In conclusion, PCB77 showed the potential to induce the expression of proinflammatory cytokines including IL-6, which has been shown to be powerful independent risk predictor of CVD. And PCB77 was observed to alter insulin-activated Akt signaling by $\mathrm{TNF} \alpha$ secretion for the first time.
\end{abstract}

Key words: PCB77; pro-inflammatory cytokines; IL-6; TNFa; NFkB; Akt

DOI: $10.1016 /$ S1001-0742(09)60221-7

\section{Introduction}

Polychlorinated biphenyls (PCBs) are a class of polychlorinated aromatic hydrocarbons composed of 209 discrete congeners. Due to their high lipophilicity and structural stability, PCBs are persistent environmental pollutants. Substantial evidence from epidemiological studies suggested that cardiovascular diseases are linked to environmental pollution. For example, there was a significant increase in mortality from cardiovascular disease among Swedish manufacturing workers exposed to PCBs for at lease five years, and most excess deaths were due to cardiovascular disease in workers exposed to phenoxy herbicides and PCBs in waste transformer oil (Gustavsson and Hogstedt, 1997; Hay and Tarrel, 1997). Epidemiology studies have also reported that the risk of insulin resistance and type 2 diabetes correlated with the serum PCBs concentration when people are exposed to this kind of pollutants (Everett et al., 2007; Rignell-Hydbom et al., 2007; Rylander et al., 2005).

Development of atherosclerotic cardiovascular disease (CVD) is the main complication in type 2 diabetes mellitus, and clinical CVD can also precede the diabetes development, leading to a hypothesis that type 2 diabetes and CVD share common antecedents (Stern, 1995). Insulin

\footnotetext{
* Corresponding author. E-mail: avaecn@gmail.com
}

resistance has been considered as a plausible candidate for this common antecedent, but mechanisms whereby insulin resistance leads to both type 2 diabetes and CVD remain unsettled (Ginsberg, 2000). Chronic subclinical inflammation could be a unifying mechanistic factor because it is a precursor of CVD, is associated with insulin resistance, and precedes the development of type 2 diabetes (Pickup, 2004). Acute-phase reactants, like C-reactive protein and their chief inductor, the proinflammatory cytokine interleukin 6, have been shown to be powerful independent risk predictors of both type 2 diabetes and CVD (Pradhan et al., 2001). Endothelial dysfunction is regarded as a causal factor in the development of atherothrombotic disease and can be detected clinically in people at risk for atherosclerosis. Endothelial dysfunction is also found in subjects with insulin resistance, in type 2 diabetes patients and in their first-degree relatives and independently predicts the future development of type 2 diabetes (Meigs et al., 2004). A specific mechanism whereby inflammation may contribute to these disease processes is induction of endothelial dysfunction, placing the vascular endothelium in a key unifying position for the shared pathogenesis of CVD and type 2 diabetes.

The present study was designed to use human umbilical vascular endothelia cell line (HUVEC cells) as studying model to investigate the pro-inflammatory reaction and 
insulin-activated signaling alteration induced by PCBs, attempting to find clues of mechanisms about the relationship of PCBs with the atherosclerotic cardiovascular disease and type 2 diabetes. From our results, PCB77 showed the potential to induce the expression of proinflammatory cytokines including IL-6, which has been shown to be powerful independent risk predictor of CVD. And PCB77 was observed to alter insulin-activated Akt signaling by TNF $\alpha$ secretion for the first time.

\section{Materials and methods}

\subsection{Cell culture and treatment}

Human umbilical vascular endothelial cells (HUVEC) and human macrophage (U937) cells were obtained from National Laboratory of Protein Engineering and Plant Genetic Engineering, College of Life Sciences, Peking University, China. The HUVEC cells were maintained in DMEM supplemented with $10 \%$ FBS as well as 100 $\mathrm{IU} / \mathrm{mL}$ penicillin and $50 \mathrm{mg} / \mathrm{mL}$ streptomycin. The U937 cells were maintained in RPM1640 supplemented with $10 \% \mathrm{FBS}$ as well as $100 \mathrm{IU} / \mathrm{mL}$ penicillin and $50 \mathrm{mg} / \mathrm{mL}$ streptomycin. The cell lines were both cultured at $37^{\circ} \mathrm{C}$ in an incubator maintained in $5 \% \mathrm{CO}_{2}$.

For cell treatment, 2,2',4,4',5,5'-hexachlorobiphenyl (PCB153; Accustandard) and 3,3',4,4'-tetrachlorobiphenyl (PCB77; Accustandard) were dissolved in dimethyl sulfoxide (DMSO) prior to addition in cell culture medium. Data has shown that serum concentration of PCBs can reach approximately $3.4 \mu \mathrm{mol} / \mathrm{L}$ after acute exposure to PCBs (Jensen, 1987). However, local microenvironmental levels of PCBs in the extracellular space are unknown. Therefore, in the present study, cells were treated with specific PCBs at the concentration of 3.4 and $5 \mu \mathrm{mol} / \mathrm{L}$. Treatment at these concentrations did not show cytotoxic effects over the time course of the experiments as determined by the MTT conversion assay (data not shown).

\subsection{Transient transfection and luciferase assay}

Transient transfection and luciferase assay were employed to study the effect of PCBs on the transcriptional activity of nuclear factor $\kappa B$. HUVEC cells were seeded at a concentration of $1 \times 10^{5}$ cells $/ 35 \mathrm{~mm}$ dish. After $12 \mathrm{hr}$, medium was changed from complete medium to DMEM without FBS or antibiotic. Transfection was done using LipofectAMINE reagent (Gibco BRL, USA) mixed with pNFkB-luc and the control vector SV40 for $8 \mathrm{hr}$ according to the manufacturers' protocol. Cells were then incubated with specific concentration of PCB77 or PCB153. The Dual-Luciferase reporter assay system (Promega) was then used for luciferase assay according to the manufacturers' protocol (Promega, USA).

\subsection{Reverse transcription and polymerase chain reac- tion (RT-PCR)}

RT-PCR was performed as previously described (Wang et al., 2008). Specifically, HUVEC cells were washed twice with PBS, and total RNA was isolated with Trizol reagent (Invitrogen, USA). cDNA first strand was formed by using SuperScript III reverse transcriptase (Invitrogen, USA) according to the manufacturer's instructions. And then polymerase chain reaction (PCR) was done for determination of the target genes using specific primers (Table 1).

Table 1 Specific amplification profiles of different target genes determination

\begin{tabular}{|c|c|c|c|}
\hline $\begin{array}{l}\text { Gene } \\
\text { name }\end{array}$ & & Primer sequence & $\begin{array}{l}\text { Product } \\
\text { size (bp) }\end{array}$ \\
\hline \multirow[t]{2}{*}{ IL-1 $\beta$} & Forward & 5'-CTGAGGAAGATGCTGGTT-3' & 373 \\
\hline & Reverse & 5'-GCTGTAGAGTGGGCTTATC-3' & \\
\hline \multirow[t]{2}{*}{ IL-6 } & Forward & 5'-TGACCCAACCACAAATGC-3' & 430 \\
\hline & Reverse & 5'-CTGGCTCTGAAACAAAGGAT-3' & \\
\hline \multirow[t]{2}{*}{ TNF $\alpha$} & Forward & $5^{\prime}$-TGGTATGAGCCCATCTATCT-3' & 294 \\
\hline & Reverse & 5'-GAAGTGGTGGTCTTGTTGC-3' & \\
\hline \multirow[t]{2}{*}{ MCP-1 } & Forward & $5^{\prime}$-CAGCCAGATGCAATCAATGC-3' & 204 \\
\hline & Reverse & 5'-GTGGTCCATGGAATCCTGAA-3' & \\
\hline \multirow[t]{2}{*}{ Actin } & Forward & $5^{\prime}$-GTGGACATCCGCAAAGAC-3' & 302 \\
\hline & Reverse & 5'-AAAGGGTGTAACGCAACTAA-3' & \\
\hline
\end{tabular}

\subsection{Western blot}

Cells were lysed with lysis buffer $(50 \mathrm{mmol} / \mathrm{L}$ Tris-HCl (pH 7.5), $150 \mathrm{mmol} / \mathrm{L} \mathrm{NaCl}, 1 \mathrm{mmol} / \mathrm{L}$ EDTA, $1 \%$ Nonidet P-40, $0.1 \mathrm{mg} / \mathrm{mL}$ leupeptin, $1 \mathrm{mmol} / \mathrm{L}$ pheylmethylsulfonyl fluoride, and $1 \mathrm{mmol} / \mathrm{L}$ sodium orthovanadate). After centrifugation at $12,000 \times g$ for $20 \mathrm{~min}$ at $4{ }^{\circ} \mathrm{C}$, supernatant was separated on SDS-PAGE. Total proteins were transferred onto PVDF membrane after electrophoresis. Western blot assay was performed with specific antibodies against phospho-Akt on Ser473 ( $\alpha$-phospho-Akt) and total Akt.

\subsection{Adhesion assay}

Adhesion was evaluated using the human leukemia promonotypic U937 cells. The HUVEC endothelial cells of 1 $\times 10^{5}$ were plated in 24 wells tissue culture plates with $1 \mathrm{~mL}$ of supplemented DMEM medium. After HUVEC endothelial cells were treated with PCB153 or PCB77 for $3 \mathrm{hr}$, cells were then washed twice with PBS and cocultivated with $5 \times 10^{5}$ U937 cells/well for $3 \mathrm{hr}$. The wells were washed to eliminate suspending U937 cells and then cells were photographed to evaluate the number of U937 adhesive to HUVEC.

\section{Results and discussion}

\subsection{Inflammatory response induced by PCB77}

PCB77 is a representative example of coplanar PCBs with dioxin-like activity, whereas PCB153 is non-coplanar but most abundant in human serum. Therefore, PCB77 and PCB153 were selected in this study. Proinflammatory cytokines (TNF $\alpha$, IL-6 and IL-1 $\beta$ ) and chemokine (MCP-1) were detected by RT-PCR. Results showed that PCB77 significantly induced IL-6, TNF $\alpha$, IL- $1 \beta$ and MCP1 gene expression, while PCB153 had minor effects on these cytokines except for IL-1 $\beta$ (Fig. 1a, b). The different induction profile by PCB77 and PCB153 has been 
a

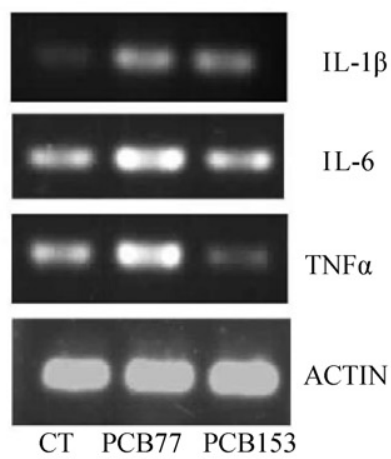

c

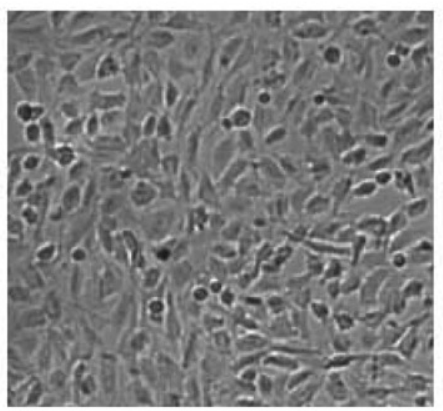

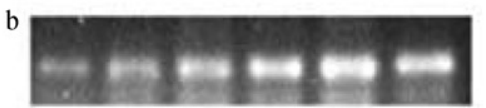

MCP-1

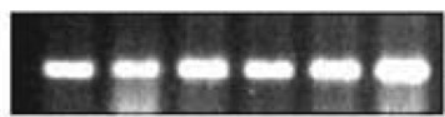

$\begin{array}{llllll}\text { CT } & 1 & 2.5 & 5 & 10 & 20\end{array}$

PCB77 $(\mu \mathrm{mol} / \mathrm{L})$

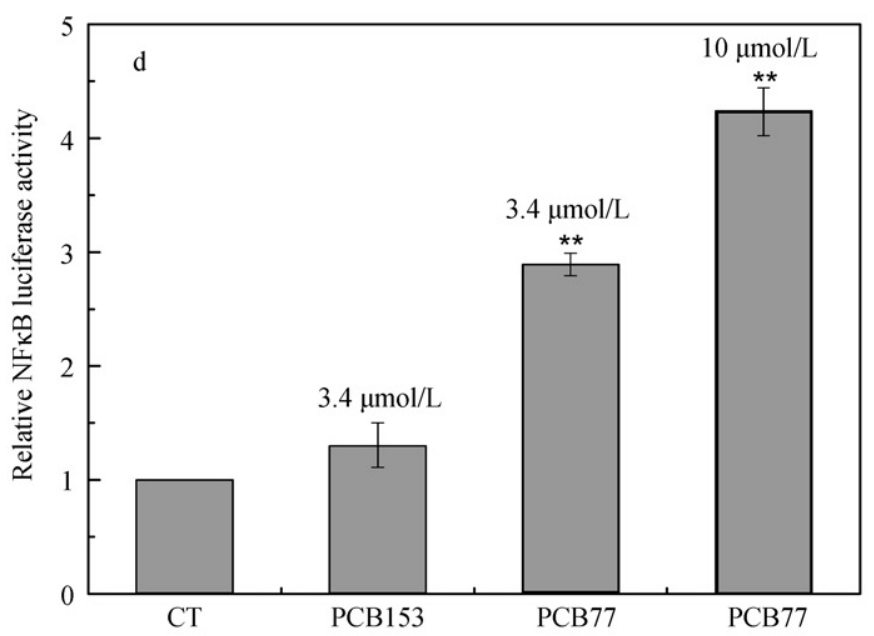

Fig. 1 Pro-inflammatory response induced by PCB77 ( $\mathrm{a}$ and $\mathrm{b}$ ) induction of inflammation cytokines by PCBs treated in HUVEC cells. (a) cells were treated by specific PCB (PCB153 and PCB77) at concentrations of $3.4 \mu \mathrm{mol} / \mathrm{L}$ for $16 \mathrm{hr}$. Induction profile of different pro-inflammatory cytokine after PCBs treatment was obtained by RT-PCR; (b) cells were treated by PCB77 at different concentrations as indicated. MCP-1 expression profile was obtained by RT-PCR. The results were shown as a representative experiment of three independent assays; (c) effect of PCBs on U937 cells adhesion to HUVEC. U937 cells were co-cultivated for $3 \mathrm{hr}$ with endothelial HUVEC cells plated in 24 well plates, pretreated with or without $5 \mu \mathrm{mol} / \mathrm{L}$ PCB153 or PCB77 for $16 \mathrm{hr}$. After removal of the suspending U937 cells from the culture plates, photographic view of U937 adhesive to HUVEC cells were obtained. Round cells represent U937 cells adhered to HUVEC. The results shown as a representative experiment of three independent assays; (d) NFKB transcriptional activation by PCBs. HUVEC cells were transfected with NFKB reporter plasmids using Lipofectamine 2000. Cells were then treated with specific concentration of PCB153 and PCB77 for $16 \mathrm{hr}$ and then reporter assay was performed. Each column represents the mean with SEM $(n=3)$ The differences between means were analyzed by one-way ANOVA. An asterisk denotes a significant difference compared with control. $* * P<0.01$. CT: control.

previously verified by other researchers although the specific cytokines detected were different from ours (Hennig et al., 2002). In that study, endothelial cell exposure to all coplanar PCBs (PCB77, PCB126, PCB169), but not to the non-AhR agonist PCB153, resulted in an activation of $\mathrm{NF \kappa B}$ and increased the production of IL-6 (Hennig et al., 2002). And IL-6 induction by PCB77 was also consistent with the results obtained in porcine pulmonary endothelial cells (Ramadass et al., 2003). As we know, induction of proinflammatory genes plays an important role in the physiological and pathological functions of the vascular endothelium. Especially, IL-6 has shown to be powerful independent risk predictor of CVD (Patterson et al., 2009).

On the other hand, increased expression of chemokines, such as monocyte chemoattractant protein-1 (MCP-1) may play a critical role in the biology of vascular dysfunction, including early phases of atherosclerosis. Result indicated that PCB77 induced MCP-1 expression in dose-dependent manner (Fig. 1b). Up-regulation of endothelial MCP-1 by PCB77 has been recently verified by Majkova et al. 
(2009), who considered that the increase of MCP-1 by PCB77 is caveolin-1-dependent. But the adhesion assay has not yet been detected. MCP-1 has known to be an endothelium-derived chemokine that attracts monocytes into sub-endothelial space in early stages of the atherosclerosis development (Hennig et al., 2002). Therefore, we performed cell adhesion assay to verify this phenomenon using co-culture system of U937 and HUVEC cells. After adherent HUVEC cells were treated with $5 \mu \mathrm{mol} / \mathrm{L}$ PCB153 and PCB77 for $16 \mathrm{hr}$, the suspending U937 cells were added and co-cultured with HUVEC cells for $3 \mathrm{hr}$. HUVEC cells and adhesive U937 cells were then photographed following removal of the suspending U937 cells by wash. Results showed that PCB77 but not PCB153 could remarkably induce U937 adhesion to HUVEC cells (Fig. 1c). The results were consistent with that of HUVEC cells treated with TNF $\alpha$ (Gutierrez et al., 2007), suggesting the pro-inflammatory cytokines, such as TNF $\alpha$ may not be excluded as precursors that cause adhesion except for MCP-1. Specific mechanisms that PCB77 attracts monocytes into sub-endothelial space need further investigation.

Finally, as a transcription factor, nuclear factor- $\kappa \mathrm{B}$ $(\mathrm{NF}-\mathrm{KB})$ plays a significant role in the induction of inflammatory responses, including regulation of inflammatory cytokine and adhesion molecule production. Binding sites for $\mathrm{NF}-\kappa \mathrm{B}$ and related transcription factors were identified in the promoter regions of a variety of inflammatory genes such as interleukin 6 (IL-6), vascular cell adhesion molecule-1 (VCAM-1) and cyclooxygenase-2 (COX-2), which have shown to be up-regulated during PCBs toxicity in the present and previous studies (Hennig et al., 2002; Kwon et al., 2002). To further investigate the mechanisms of PCBs inducing endothelial dysfunction, NFKB activity was assayed in HUVEC cells after the treatment with PCB77 and PCB153. Consistent with the cytokine induction profile, PCB77 showed a significant activation of $\mathrm{NF \kappa B}$ transcription in dose-dependent manner, while PCB153 could not induce NFKB activation (Fig. 1d), suggesting NF- $\mathrm{NB}$ an important factor to induce inflammatory responses.

\subsection{Insulin signaling alteration induced by PCB77}

Because endothelial dysfunction has been found in subjects with insulin resistance and type 2 diabetes patients, its related mechanisms becomes a new target to investigate insulin resistance and diabetes (Pradhan et al., 2001; Andreozzi et al., 2007; Monroy et al., 2009). Using HUVEC cells as model, we therefore investigate whether PCB77 alter the insulin-activated signaling, while it has induced significant inflammation response in endothelial cells. From the results, we found that Akt signaling was activated represented by phosphorylation of Akt on $\mathrm{Ser}^{473}$ when HUVEC cells were incubated with insulin only. However, PCB77 treatment blocked insulin-activated Akt signaling (Fig. 2). Considering the possible effects of proinflammatory cytokines on the proinflammation response and adhesive induction, we performed the same assay pretreated with different cytokine neutralization antibodies. Results showed that TNF $\alpha$ neutralization antibodies could

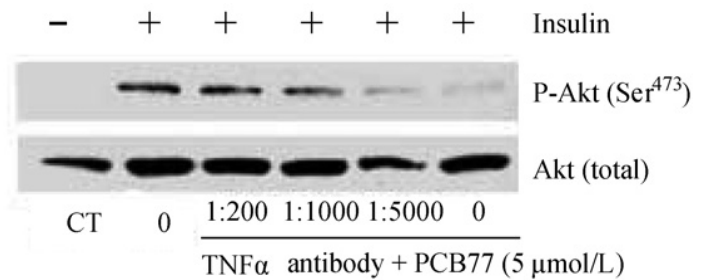

Fig. 2 Insulin signaling alteration induced by PCB77. HUVEC cells were pretreated with or without $\mathrm{TNF} \alpha$ neutralization antibody followed by PCB77 incubation ( $5 \mu \mathrm{mol} / \mathrm{L})$ for $16 \mathrm{hr}$, cells were then activated by insulin $(100 \mathrm{nmol} / \mathrm{L})$ for $30 \mathrm{~min}$. Total cell lysates were then separated by SDS-PAGE and immuno-blotted with antibodies against phospho-Akt on $\operatorname{Ser}^{473}$ ( $\alpha$-phospho-Akt) and total Akt. The results were shown as a representative experiment of three independent assays.

remarkably restore insulin-activated Akt signaling blocked by PCB77 (Fig. 2). These data showed that alteration of insulin signaling by PCB77 may be due to the proinflammatory cytokine, especially $\mathrm{TNF} \alpha$ induction. This is the first study suggesting that PCB77 block the insulinactivated Akt by TNF $\alpha$ secrection. Researchers have found that proinflammatory cytokine including IL- 6 and TNF $\alpha$ could impair the insulin signaling pathway (Andreozzi et al., 2007; Monroy et al., 2009). IL-6 is likely to be mediated by the activation of JNK and ERK1/2 followed by phosphorylation of IRS-1 (Andreozzi et al., 2007). The membrane proteolysis regulation of TNF $\alpha$ converting enzyme (TACE) and its inhibitor, tissue inhibitor of metalloproteinase 3 (TIMP3) may be an important factor for the development of skeletal muscle insulin resistance in obese type 2 patient (Monroy et al., 2009). In our study, TNF $\alpha$ not IL-6 is necessary in the blocked insulin signaling by PCB77. Further studies are needed to investigate the specific mechanisms that PCB77 alters insulin signaling.

\section{Conclusions}

In the present study, we found that PCB77 had the potential to induce the expression of proinflammatory cytokines including IL-6, which has been shown to be powerful independent risk predictor of CVD, and PCB77 could alter insulin-activated signaling by the secretion of $\mathrm{TNF} \alpha$. Therefore, PCB77 may provide a new contribution to be indicated risk factor that cause both atherosclerosis and insulin resistance and type 2 diabetes.

\section{Acknowledgments}

This work was supported by the National Basic Research Program (973) of China (No. 2009CB421605) and the National Natural Science Foundation of China (No. 20890112).

\section{References}

Andreozzi F, Laratta E, Procopio C, Hribal M L, Sciacqua A, Perticone $\mathrm{M}$ et al., 2007. Interleukin-6 impairs the insulin signaling pathway, promoting production of nitric oxide in human umbilical vein endothelial cells. Molecular and Cellular Biology, 27: 2372-2382. 
Everett C J, Frithsen I L, Diaz V A, Koopman R J, Simpson W M Jr, Mainous A G, 2007. Association of a polychlorinated dibenzo-p-dioxin, a polychlorinated biphenyl, and DDT with diabetes in the 1999-2002. In: National Health and Nutrition Examination Survey (3rd ed.). Environmental Research, 103: 413-418.

Ginsberg H N, 2000. Insulin resistance and cardiovascular disease. The Journal of Clinical Investigation, 106: 453-458.

Gustavsson P, Hogstedt C, 1997. A cohort study of Swedish capacitor manufacturing workers exposed to polychlorinated biphenyls (PCBs). American Journal of Industrial Medicine, 32: 234-239.

Gutierrez G, Mendoza C, Zapata E, Montiel A, Reyes E, Montano L F et al., 2007. Dehydroepiandrosterone inhibits the TNFalpha-induced inflammatory response in human umbilical vein endothelial cells. Atherosclerosis, 190: 90-99.

Hay A, Tarrel J, 1997. Mortality of power workers exposed to phenoxy herbicides and polychlorinated biphenyls in waste transformer oil. Annals of the New York Academy of Sciences, 837: 138-156.

Hennig B, Meerarani P, Slim R, Toborek M, Daugherty A, Silverstone A E et al., 2002. Proinflammatory properties of coplanar PCBs: in vitro and in vivo evidence. Toxicology and Applied Pharmacology, 181: 174-183.

Jensen A A, 1987. Polychlorobiphenyls (PCBs), polychlorodibenzo- $p$-dioxins (PCDDs) and polychlorodibenzofurans (PCDFs) in human milk, blood and adipose tissue. Science of the Total Environment, 64: 259-293.

Kwon O, Lee E, Moon $\mathrm{T}$ C, Jung $\mathrm{H}$, Lin C X, Nam $\mathrm{K}$ S et al., 2002. Expression of cyclooxygenase-2 and pro-inflammatory cytokines induced by $2,2^{\prime}, 4,4^{\prime}, 5,5^{\prime}$ hexachlorobiphenyl (PCB 153) in human mast cells requires NF-kappa B activation. Biological and Pharmaceutic Bulletin, 25: 1165-1168.

Majkova Z, Smart E, Toborek M, Hennig B, 2009. Up-regulation of endothelial monocyte chemoattractant protein-1 by coplanar PCB77 is caveolin-1-dependent. Toxicology and Applied Pharmacology, 237: 1-7.

Meigs J B, Hu F B, Rifai N, Manson J E, 2004. Biomarkers of endothelial dysfunction and risk of type 2 diabetes mellitus. The Journal of the American Medical Association, 291: 1978-1986.

Monroy A, Kamath S, Chavez A O, Centonze V E, Veerasamy M, Barrentine A et al., 2009. Impaired regulation of the TNF-alpha converting enzyme/tissue inhibitor of metalloproteinase 3 proteolytic system in skeletal muscle of obese type 2 diabetic patients: A new mechanism of insulin resistance in humans. Diabetologia, 52: 2169-2181.

Patterson C C, Smith A E, Yarnell J W, Rumley A, Ben-Shlomo Y, Lowe G D, 2010. The associations of interleukin-6 (IL-6) and downstream inflammatory markers with risk of cardiovascular disease: the caerphilly study. Atherosclerosis, 209: 551-557.

Pickup J C, 2004. Inflammation and activated innate immunity in the pathogenesis of type 2 diabetes. Diabetes Care, 27: 813-823.

Pradhan A D, Manson J E, Rifai N, Buring J E, Ridker P M, 2001. C-reactive protein, interleukin 6, and risk of developing type 2 diabetes mellitus. The Journal of the American Medical Association, 286: 327-334.

Ramadass P, Meerarani P, Toborek M, Robertson LW, Hennig B, 2003. Dietary flavonoids modulate PCB-induced oxidative stress, CYP1A1. Induction, and AhR-DNA binding activity in vascular endothelial cells. Toxicological Sciences, 76: 212-219.

Rignell-Hydbom A, Rylander L, Hagmar L, 2007. Exposure to persistent organochlorine pollutants and type 2 diabetes mellitus. Human and Experimental Toxicology, 26: 447452.

Rylander L, Rignell-Hydbom A, Hagmar L, 2005. A crosssectional study of the association between persistent organochlorine pollutants and diabetes. Environmental Health, 4: 28.

Stern M P, 1995. Diabetes and cardiovascular disease. The "common soil" hypothesis. Diabetes, 44: 369-374.

Wang J, Wu S, Jin X, Li M, Chen S, Teeling J L et al., 2008. Retinoic acid-inducible gene-I mediates late phase induction of TNF-alpha by lipopolysaccharide. The Journal of Immunology, 180: 8011-8019. 